\title{
Correction to: School Security Measures and Longitudinal Trends in Adolescents' Experiences of Victimization
}

\author{
Benjamin W. Fisher $\mathbb{1}^{1} \cdot$ Thomas J. Mowen ${ }^{2} \cdot$ John H. Boman IV
}

Published online: 13 July 2020

(c) Springer Science+Business Media, LLC, part of Springer Nature 2020

Correction to: Journal of Youth and Adolescence (2018) 47:1221-1237

https://doi.org/10.1007/s10964-018-0818-5

The original version of the article was inadvertently published with errors in the text and Table 2. The corrected text and the table are given below:

In section "School Security", third para, page 1225, the sentence should read, "As a result, the final construct is constructed of: (a) controlled access to the school grounds, (b) use of a closed campus for lunch, (c) presence of drug sniffing dogs, (d) strict dress code, (e) required ID badges for students, (f), required ID badges for faculty/staff, (g) security cameras to monitor students, (h) panic buttons in classrooms, and (k) presence of security guards. Descriptive statistics for this measure - designed to have a mean of 0 and a standard deviation of 1 -are presented in Table 2.

Note that these changes do not affect the regression models.

Publisher's note Springer Nature remains neutral with regard to jurisdictional claims in published maps and institutional affiliations.
The original article can be found online at https://doi.org/10.1007/ s10964-018-0818-5.

Benjamin W. Fisher

ben.fisher@louisville.edu

$\triangle$ Thomas J. Mowen

tmowen@bgsu.edu

$\triangle$ John H. Boman

jboman@bgsu.edu

1 Department of Criminal Justice, University of Louisville, 2301 South Third Street, Louisville, KY 40205, USA

2 Department of Sociology, Bowling Green State University, Bowling Green, $\mathrm{OH} 43403$, USA 
Table 2 Two parameter logistic item response theory results for school security measures

\begin{tabular}{|c|c|c|}
\hline Security Measure & Coef. & $S E$ \\
\hline \multicolumn{3}{|c|}{ Controlled Access to School Grounds } \\
\hline Discrimination & 0.670 & $0.026 * * *$ \\
\hline Difficulty & 0.484 & $0.032 * * *$ \\
\hline \multicolumn{3}{|c|}{ Closed Campus for Lunch } \\
\hline Discrimination & 0.438 & $0.026 * * *$ \\
\hline Difficulty & -1.948 & $0.115^{* * *}$ \\
\hline \multicolumn{3}{|l|}{ Drug Sniffing Dogs } \\
\hline Discrimination & 0.462 & $0.234 * * *$ \\
\hline Difficulty & 0.617 & $0.048 * * *$ \\
\hline \multicolumn{3}{|l|}{ Strict Dress Code } \\
\hline Discrimination & 0.172 & $0.022 * * *$ \\
\hline Difficulty & -1.543 & $0.221 * * *$ \\
\hline \multicolumn{3}{|c|}{ Require ID Badges for Students } \\
\hline Discrimination & 2.705 & $0.112 * * *$ \\
\hline Difficulty & 1.202 & $0.022 * * *$ \\
\hline \multicolumn{3}{|c|}{ Require ID Badges for Faculty/Staff } \\
\hline Discrimination & 2.824 & $0.123 * * *$ \\
\hline Difficulty & 0.375 & $0.013 * * *$ \\
\hline \multicolumn{3}{|l|}{ Security Cameras } \\
\hline Discrimination & 0.724 & $0.027 * * *$ \\
\hline Difficulty & 0.148 & $0.027 * * *$ \\
\hline \multicolumn{3}{|c|}{ Panic Buttons in Classroom } \\
\hline Discrimination & 0.413 & $0.024 * * *$ \\
\hline Difficulty & -0.365 & $0.047 * * *$ \\
\hline \multicolumn{3}{|l|}{ Security Guards } \\
\hline Discrimination & 1.193 & $0.039 * * *$ \\
\hline Difficulty & -0.637 & $0.023 * * *$ \\
\hline
\end{tabular}

